\title{
Optimization and performance assessment of Solar Towers
}

\author{
Elisa Ghirardi $^{1 *}$, Giovanni Brumana ${ }^{1}$, and Giuseppe Franchini ${ }^{1}$ \\ ${ }^{1}$ University of Bergamo, Department of Engineering and Applied Sciences, 5 Marconi Street, 24044 \\ Dalmine, Italy
}

\begin{abstract}
The present paper investigates possible strategies to improve the competitiveness of Solar Towers, considered the best option over CSP technologies. Nevertheless, many aspects still penalize the tower systems, mainly the higher installation costs and the lower energy density. The optimal design of the heliostat layout and the selection of the optimal tower height are fundamental to improve the performance of CRS. A new model for optimizing and simulating solar tower plants, based on an in-house Matlab ${ }^{\circledR}$ code, has been developed and validated. A technical and an economic optimization procedure allows to select the plant configuration with the maximum efficiency or the minimum LCOE, respectively. The case study is focused on a solar field of 6000 heliostats, corresponding to a nominal power of $100 \mathrm{MWe}$. The tower height shows a strong influence on the heliostat layout and solar field performance; however, the annual energy yield shows a nearly asymptotic behavior when the tower height is increased. An economic optimization leads to a less dense layout to limit the tower impact on the cost; a penalty in efficiency of around $6 \%$ can reduce the LCOE of more than 5\%. The minimization of land utilization, saving $24 \%$ of the occupied area, has a penalization of about $8 \%$ in terms of LCOE.
\end{abstract}

\section{Acronyms}

\begin{tabular}{|l|l|}
\hline$C S P$ & Concentrated solar power \\
\hline CRS & Central receiver system \\
\hline$D N I$ & Direct normal irradiance \\
\hline EPC & Engineering, Procurement \& Construction \\
\hline$H T F$ & Heat transfer fluid \\
\hline LCOE & Levelized cost of electricity \\
\hline OM & Operation and maintenance \\
\hline$P T C$ & Parabolic trough collector \\
\hline$T H T$ & Total tower height \\
\hline
\end{tabular}

\footnotetext{
* Corresponding author: elisa.ghirardi@unibg.it
} 


\section{Introduction}

In recent years, the solar thermal plant are increasingly used to supply the variability and the non-predictable nature of other renewable technologies more developed, such as photovoltaic and wind [1]. The higher operative temperature level of Solar Power Towers (also called Central Receiver Systems, CRS) over the others CSP technologies suggests a greatest development potential [2].

The competitiveness of the CRS compared to the PTC is mostly penalized by the land occupancy and by the cost of the plant. The utility-scale applications help to limit the cost rising [3], while the two tracking axis system ensure a more constant seasonal behavior and a greater load following capability is achievable [4]. Many studies have demonstrated that improve the heliostat efficiency can enhance by $10 \%$ the energy delivered by the field [5]. The effect of the plant design parameters on the system performance are still unclear, as underlined by Rui Chen et al. [6], such as the design meteorological condition or the solar multiple. Recently different authors addressed the field optimization from different points of view. Gadalla and Sagafifar establish the best heliostat arrangement based on the shadows and block efficiency [7]. Luo et al. demonstrate that the heliostat layout and the receiver are independent and the optimization of the CRS system could be split in two separated procedures [8]. Schöttl et al. prefer a whole field approach to the performance evaluation of individual heliostat, the layout boundaries are determined with consideration on annual efficiency and land occupancy [9].

Moreover, the influence of the design parameters changes with different optimization function. Li et al. demonstrated that the most efficient solution is not the cheapest one [10]. Leonardi et al. analyzed the impact of the land cost on the layout of a $55 \mathrm{MW}$ plant, underlying that is possible to reduce the area by almost $50 \%$ without damaging the annual efficiency [11]. Kiwan and Hamad considered the tilt of the land to minimize the occupancy, however the resulting annual efficiency is damaged [12].

An additional issue is introduced when the size of the plant increases. In the maximization of the power collected on the receiver, Cruz et al. underlines that managing a commercial scale heliostat field means an high computationally optimization cost [13]. Collado and Guallar in [14] extend their field analysis to a commercial scale CRS plant (more than 100 $\mathrm{MWe}$ ) and it is founded out that the impact of strong layout changes is minimal for fixed receiver and tower dimensions.

In the present work the influence of the tower height on the layout displacement is analyzed in detail, in particular on the heliostat spacing, for a large-scale plant. Furthermore, the influence of the optimization function on techno-economic performance of the resulting layout is analyzed; four different objective are imposed: the maximization of the design efficiency and annual efficiency, the minimization of the cost of electricity (LCOE) and the minimization of the land usage.

\section{Material and methods}

The flexibility of the CRS plant production could be managed with a different design strategy. This study has the objective to determine the influence on the performance and the cost of a utility-scale tower plant, with fixed heliostat number Nhel, when the objective function imposed in the optimization process is varied. Firstly, the model for the generation and the simulation of the solar field is presented; then the different optimization approaches are analyzed. 


\subsection{Model description}

A radial staggered surrounding heliostat field, coupled with an external cylindrical molten salt receiver, has been selected in this work since it shown to be the most competitive configuration for electric production applications [15]. Following, the procedure to generate a regular radially staggered layout is resumed briefly: the heliostats are organized in concentric rings and the algorithm changes the radial distance between consecutive rows as function of the parameter $d R$ and the heliostat size $D M$, starting from the minimum spacing that ensure the no-touching condition $\left(d R_{\min }=0.866 \cdot D M\right)($ Eq. 1$)$. The field is divided into regions, called groups, in which the azimuthal spacing $\Delta \alpha$ between heliostats in the same row is kept constant: a new group is started when the number of heliostats per row can be doubled with respect to the space limit imposed by $D M$. The adopted spacing criterion in used to maximize the field density in the first three groups of the field, whilst the last zone will be generated by the staggered method for a better shading and blocking efficiency [16]. To maximize the yield efficiency, the radial increment in the group $\Delta R_{i}$ is kept constant, as shown by Collado and Guallar [17].

$$
\begin{aligned}
& \Delta R_{i}=d R_{i} \cdot D M \\
& \Delta \alpha_{i}=2 \operatorname{asin}\left[D M / 2 R_{i}\right] \cong D M / R_{i} \\
& R_{1}=0.75 \cdot T H T
\end{aligned}
$$

The radius of the first ring is determined as a function of tower height THT (Eq. 3) to maximize the efficiency, as demonstrated by [18], and the number of heliostat per ring is then fixed.

To mitigate the rigidity resulting from the pattern layout, a bigger field is generated according to Eq. 1 and Eq. 2; the optical efficiency of each heliostat $\eta_{\text {helio }}$ is evaluated on reference condition, according to the Eq. 4 [19], and the final layout selects the more efficient mirrors. Given the suggestion of Wang [16], after the heliostats selection a second simulation run has been carried out, to avoid the influence of the deleted ones. The field efficiency $\eta_{\text {field }}$ is the average of the efficiencies of the mirrors in the final layout.

$$
\eta_{\text {helio }}(x, y, t)=\rho(t) \cdot f_{\text {cos }}(x, y, t) \cdot f_{\text {att }}(x, y, t) \cdot f_{\text {spill }}(x, y, t) \cdot f_{s b}(x, y, t)
$$

The main optical parameters included in the efficiency model adopted in this work (eq.4) are presented below:

- $\quad$ the mirror reflectivity $\rho$, assumed to be constant and equal to 0.9.

- The cosine effect $f_{\text {cos }}$ represents the cosine of the angle between the incident sun rays and the normal to the heliostat.

- The atmospheric attenuation $f_{\text {att }}$ is evaluated starting from the extinction model, presented by [20], based on the length of the ray path from the heliostat to the receiver.

- The spillage factor $f_{\text {spill }}$, represents the amount of reflected energy by the heliostat that is collected by the receiver. The adopted model, based on HFLCAL method [21], is reported in eq. 5 . The effective collection boundaries correspond to receiver dimensions projected into the image plane, normal to the reflected ray (with zenith angle of $\varepsilon_{T}$ ). It is assumed that the power distribution of the reflected energy has a Gaussian distribution with a standard deviation $\sigma_{H F}$.

$$
f_{\text {spill }}(x, y, t)=\operatorname{erf}\left(\frac{R r e c}{\sigma_{H F} \sqrt{2}}\right) \frac{1}{2}\left(\operatorname{erf}\left(\frac{\left(\frac{H r e c}{2}\right) \sin \varepsilon_{T}}{\sigma_{H F} \sqrt{2}}\right)-\operatorname{erf}\left(\frac{\left(-\frac{H r e c}{2}\right) \sin \varepsilon_{T}}{\sigma_{H F} \sqrt{2}}\right)\right)
$$

- The shadowing and blocking effect $f_{s b}$, compute as the reduction of the effective mirror area, considers the interference in the sun ray reflection by the other heliostats in the field. The neighboring heliostat with the higher probability to block the rays are the closer to the mirror and to the projected sun ray (from the sun o to the tower, for the shading and blocking respectively), as shown in Fig. 
1 [22]. Once identified the six candidate mirrors, their surface is projected onto the analyzed one, reducing the overestimation of the Sassi's method [23].

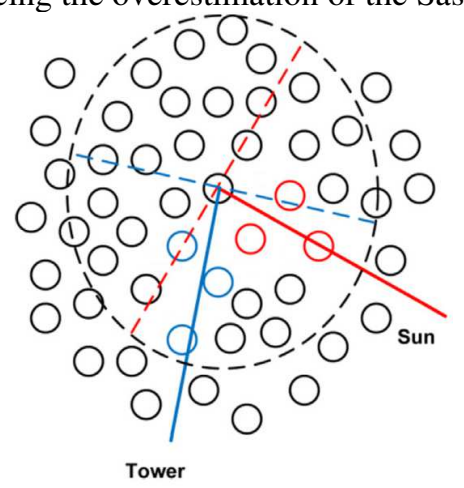

Fig. 1. Identification of the possible shading (red) and blocking (blue) heliostats.

\subsection{Optimization procedure}

A complete grid optimization of the design parameters that affect the plant performance has been made. The objective function considered in this work are:

- Maximization of the design field efficiency $\eta_{\text {field }}$, computed for the reference condition of the Solar noon of $21^{\text {st }}$ March (Eq. 6)

- Maximization of the annual efficiency $\eta_{a n n}$, calculated as the DNI weighted average efficiency during the whole year (Eq. 7).

- Minimization of the land usage $A_{\text {land }}$, determined by the convolution of the heliostat in the field and include a non-reflective area $A_{\text {non field }}$ for the system structure (Eq. 8)

Minimization of the levelized cost of electricity LCOE, normally used as metric to compare different power generation systems (Eq. 9). The annualization method is selected for the calculation of the LCOE of the plant and the operation and maintenance cost are divided by fixed and variable. All the financial parameters, reported in Table 1, and the cost model adopted (Eq 10-11) derives from SAM software [24].

$$
\begin{aligned}
& \eta_{\text {field }}=\frac{\sum_{i=1}^{N h e l} \eta_{\text {hel, },}}{\text { Nhel }} \\
& \eta_{\text {ann }}=\sum_{n=1}^{365} \frac{\int_{D N I \geq 150} D N I \cdot \eta_{\text {field }}(t) d t}{\int_{\substack{D^{\circ} \\
\alpha \geq 15^{\circ}}} D N I d t} \\
& A_{\text {land }}=A_{\text {refl }}+A_{\text {non field }} \\
& L C O E\left(U S D / k W h_{e}\right)=\frac{\frac{i(1+i)^{N y}}{(1+i)^{N y}} C_{p l a n t}+O M_{f}}{E_{e l}}+O M_{v} \\
& \text { Plant }_{\text {cost }}=C_{\text {helio }}+C_{\text {tower }}+C_{\text {rec }}+C_{P B} \\
& C_{\text {Plant }}=\text { Plant }_{\text {cost }} \cdot(1+\text { Contingency }) \cdot(1+\text { Sales }+E P C)+\text { Land }_{\text {cost }}
\end{aligned}
$$

Table 1. Specification of the financial and economic values. 


\begin{tabular}{|c|c|c|c|c|c|}
\hline \multicolumn{3}{|c|}{ Financial } & \multicolumn{3}{|c|}{ Economic } \\
\hline Variable & Value & & Component & Cost model & \\
\hline Contingency & $7 \%$ & & Heliostat & $156 \cdot$ Nhel $\cdot$ Area $a_{\text {hel }}$ & $\$ / m^{2}$ \\
\hline Sales & $4 \%$ & & Tower & $3 E 6 \exp \left(0.0113\left(T H T-H_{r e c} / 2\right)\right)$ & $\$ / m$ \\
\hline EPC & $13 \%$ & & Receiver & $1.03 E 8\left(\text { Area }_{\text {rec }} / 1571\right)^{0.7}$ & $\$ / m^{2}$ \\
\hline Discount rate $i$ & $4 \%$ & & Power block & $1.33 \cdot P_{\text {nom }}$ & $\$ / M W e$ \\
\hline OM fixed cost & 66 & \$/MW & Land & $1.25 \cdot$ Area $_{\text {land }}$ & $\$ / m^{2}$ \\
\hline OM variable cost & 0.0035 & $\$ / \mathrm{kWh}$ & & & \\
\hline Life span (years) $N_{y}$ & 35 & & & & \\
\hline
\end{tabular}

The mathematical structure of the field performance does not show any explicit dependence on the design parameters. Since each optical loss has different contribution and a trade-off must be found, the optimization process, schematized in fig.1, must include the performance evaluation of each heliostat in field. Starting from new set of variables (i.e. $d R_{-} i$ and $H_{t}$ ), a heliostat field is created according to Eq. 1-3 and the selected number of mirrors (Nhel) are chosen from the more efficient heliostat in reference condition: usually the solar noon of 21st March is preferred excepted for the maximization of the annual efficiency where the entire annual data are used. The resulting layout is then simulated on annual bases in order to determine the annual efficiency and the total energy delivered to the heat transfer fluid $E_{H T F}$. The optimization algorithm monitors the output functions and determined the best solution (tower height and layout configuration) that minimized/maximized the selected objective function.

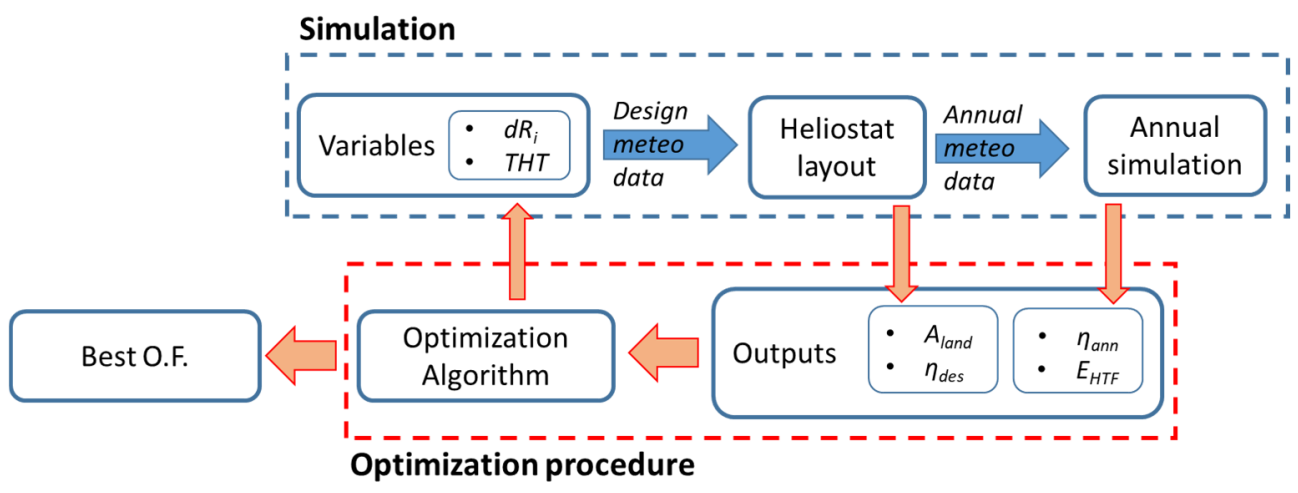

Fig. 2. Schematization of the optimization procedure.

The optimization procedure selects the combination of the design variables in the search space, reported in Table 2, with the considered step value. The radial increment analysis is limited to the second and third groups, because in the first group $d R_{1}$ is fixed as the minimum allowable value (0.866-DM), to maximize the land utilization in the most efficient region [25].

Table 2. Definition of the search space.

\begin{tabular}{ccc}
\hline Variable & Range & Step \\
\hline dR2 & $0.866-1.2$ & 0.085 \\
dR3 & $0.866-2.2$ & 0.2 \\
THT & $150-300 \mathrm{~m}$ & $10 \mathrm{~m}$ \\
\hline
\end{tabular}

\section{Results and discussion}


This study focused on the solar system optimization for a commercial scale central receiver system plant with a thermal design output of about $600 \mathrm{MWth}$, corresponding to a heliostat field with 6000 mirrors. In Table 3 the main parameters imposed to the field are reported. The receiver is not directly included in the following analysis and the dimensions considered are determined by the software SAM for a plant with the same power. The plant is assumed to be installed in Riyadh (Saudi Arabia) and the meteorological condition are provided by the Meteonorm database [26].

Table 3. Field parameters and assumption.

\begin{tabular}{lcc}
\hline & Values & Unit \\
\hline Heliostat dimensions $(l m \times w m)$ & $12.3 \times 15.36$ & $\mathrm{~m}$ \\
Heliostat mirror area $\left(\right.$ Area $\left._{\text {hel }}\right)$ & 183 & $\mathrm{~m}^{2}$ \\
Number of heliostats & 6000 & - \\
Receiver dimensions $(R H \times R D)$ & $18.4 \times 16.45$ & $\mathrm{~m}$ \\
Receiver design power & 600 & $\mathrm{MWh}$ \\
\hline
\end{tabular}

\subsection{Model validation}

The efficiency model (Eq.4), written in Matlab language, is validated against the data available in [27], even for the new approach of shadowing and blocking factor. In Table 4, the comparison of the optical terms is reported for two fields; the difference from the reference always below $1 \%$ confirm a good accuracy of the model. About the computational times, a single optimization on 8 -core processor at $2.50 \mathrm{GHz}$ and $8 \mathrm{~GB}$ of RAM requires less than $24 \mathrm{sec}$.

Table 4. Validation of the efficiency model.

\begin{tabular}{|c|c|c|c|c|c|c|}
\hline & \multicolumn{3}{|c|}{ Case 1} & \multicolumn{3}{|c|}{ Case 2} \\
\hline & Ref & New model & Diff $(\%)$ & Ref & New Model & Diff (\%) \\
\hline$f_{\cos }$ & 0.815 & 0.8164 & $-0.2 \%$ & 0.8006 & 0.8018 & $-0.1 \%$ \\
\hline$f_{a t t}$ & 0.9568 & 0.9502 & $0.7 \%$ & 0.9515 & 0.943 & $0.9 \%$ \\
\hline$f_{s b}$ & 0.9128 & 0.9184 & $-0.6 \%$ & 0.9563 & 0.9594 & $-0.3 \%$ \\
\hline$f_{\text {spill }}$ & 0.9948 & 0.9979 & $-0.3 \%$ & 0.9855 & 0.992 & $-0.7 \%$ \\
\hline
\end{tabular}

\subsection{Heliostat field layout}

First, the dependence of the radial spacing with the tower in order to maximize the efficiency is analyzed (first and second objective function). The optimization result reports that, in addition to the first group, the optimal configuration of the second group is represented by the densest solution $\left(d R_{2}=0.866 \cdot \mathrm{DM}\right)$; the main cause could be the increasing number of heliostats in the furthest region from the tower. In Fig 3a the radial space of the third group $d R_{3}$ that maximized the design (blue) or annual efficiency (green) is highlighted. For the lower tower height considered, the efficiency of the $21^{\text {st }}$ March noon allows to slightly reduce the layout expansion; when the tower is increased, no significant differences are depicted.

The layout efficiency does not highlight a clear maximum point: greater is the tower, greater is the achieved efficiency. However, a border point for a tower height of $210 \mathrm{~m}$ is observed: the improvement rate $(\Delta \eta / \Delta T H T)$ suddenly change from 0.0008 to 0.0003 .

The annual efficiency of the layouts that maximize the design efficiency is marginally lower, in the order of $0.1 \%$, than the ones that maximize the annual efficiency. The largescale layout results to be less sensitive to the design period since the number of the heliostat is huge and the single performance could be sacrificed for the whole field; reducing the 
optimization period to the noon of the $21^{\text {st }}$ March it is possible to maximize the annual weighted efficiency.

The land occupied by the field for the different tower height is reported in Fig. 3b, increasing the tower optical height the land occupied by the 6000 heliostat is always lower and it is possible to save almost $40 \%$ of land with a tower height of $300 \mathrm{~m}$, reaching a minimum value of $3.682 \mathrm{~km}^{2}$. Despite the greater expansion, the radii of the mirror rings (Eq. 3 ) are greater and more of heliostat can be placed near the tower, without damaging the efficiency.

The LCOE (Fig. 3b dotted) is a combined function: for a fixed tower height, minimize the Eq. 9 correspond to the maximization of the annual field efficiency and since the land cost is marginal, the optimal radial spacing does not change.

The minimum value of the LCOE is achieved for a THT of around $210 \mathrm{~m}$. The increase of efficiency with a greater tower height is not sufficient to compensate the greater cost of the tower. Furthermore, a greater THT correspond to a greater thermal loss along the pipe and the energy delivered to the heat transfer fluid reaches a nearly asymptotic value.

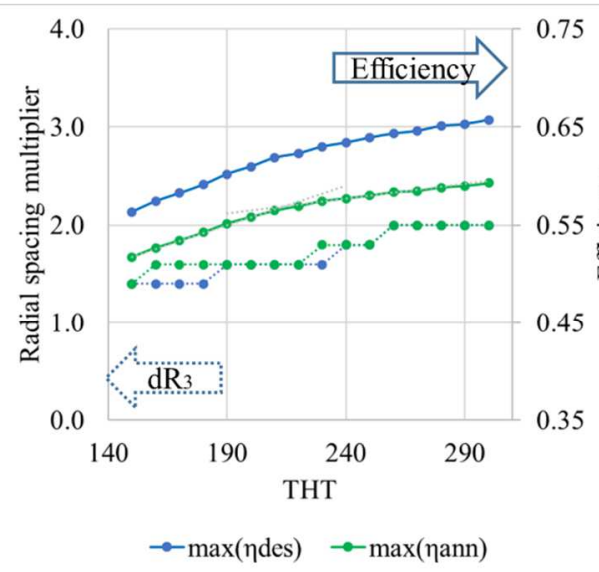

(a)

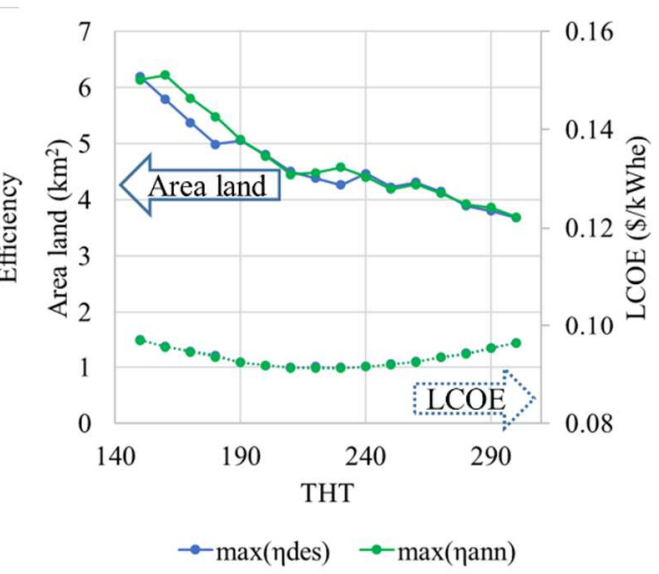

(b)

Fig. 3. Comparison of the geometrical parameter and the performance of the optimum layout that maximize the design (blue) and the annual (green) efficiency.

When the land minimization is considered, the optimal field correspond to the solution without expansions ( $d R_{i}=0.866$ in each group). In Fig. $4 \mathrm{a}$, it is highlighted that the minimal radial spacing leads to a minimal ground usage, however, the efficiency is heavily penalized meanly by the worst shadow and block factor. A penalization of around $13 \%$ is reduced to $5 \%$ only when high tower heights are considered. The annual penalization rises by $1 \%$ and this contributes to a different trend of the LCOE with respect to the one presented in fig. 3: since the low efficiency, every improvement has a greater impact than the increased cost when the tower is higher.

Changing the tower height, the dependence of the land usage is very limited (Fig. $4 \mathrm{~b}$ orange) and a value of around $2.8 \mathrm{~km}^{2}$ is reached. The solutions that maximize the efficiency (Fig. $4 \mathrm{~b}$ - blue) shows a land coverage always higher. 


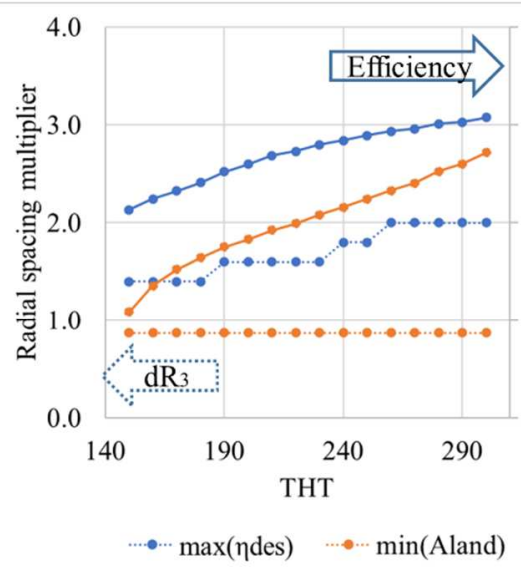

(a)
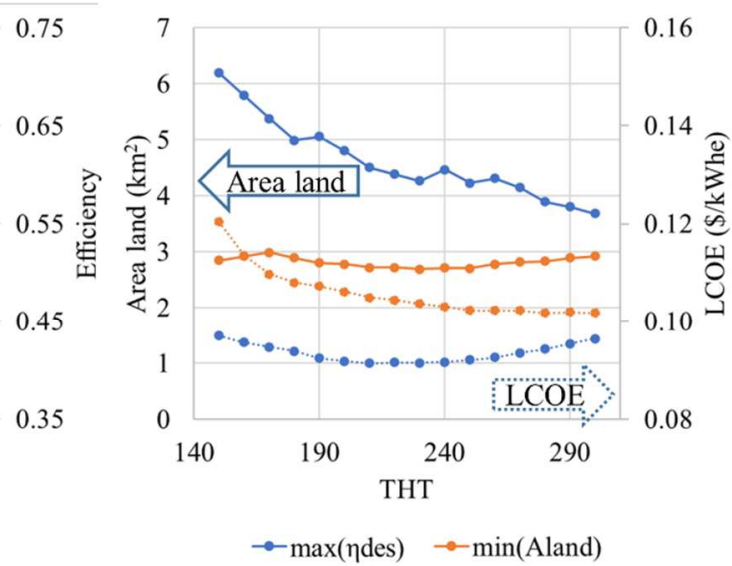

$\rightarrow \max (\eta \mathrm{des}) \quad \rightarrow \min ($ Aland $)$

(b)

Fig. 4. Comparison of the geometrical parameter and the performance of the optimum layout that maximize the design efficiency (blue) and minimize the land occupied (orange).

In Table 5, the four solution that optimize the different objective function are summarized, highlighting the efficiency, the cost of the plant and the electricity and the land occupancy. As already discussed, it is underlined that maximize the field design or the annual efficiency leads to layouts with almost the same performance. Considering the LCOE as objective function, a penalization of $5.88 \%$ in field efficiency is compensate by a saving of $5.25 \%$ of the electricity cost. More than $26 \%$ of land can be saved if the occupied area is minimized during the optimization but the efficiency and consequently the energy collected are strongly penalized. This involves in an inflation of $8 \%$ of the LCOE. Compared to the cheapest solution the area reduction is almost doubled but the cost increases by $13 \%$.

Table 5. Summary of the optimal layouts.

\begin{tabular}{ccccccc}
\hline $\begin{array}{c}\text { Objective } \\
\text { function }\end{array}$ & $\begin{array}{c}H_{\text {topt }} \\
(\mathrm{m})\end{array}$ & $\eta_{\text {des }}$ & $\eta_{\text {ann }}$ & $\begin{array}{c}\text { Plant cost } \\
(\mathrm{m})\end{array}$ & $\begin{array}{c}\text { LCOE } \\
\left(\$ / k W h_{e}\right)\end{array}$ & $\begin{array}{c}A_{\text {land }} \\
\left(\mathrm{km}^{2}\right)\end{array}$ \\
\hline$\eta_{\text {des }}$ & 300 & 0.6574 & 0.5925 & 914.241 & 0.09645 & 3.682 \\
$\eta_{\text {ann }}$ & 300 & 0.6572 & 0.5927 & 914.249 & 0.09641 & 3.688 \\
$L C O E$ & 210 & 0.6187 & 0.5643 & 813.428 & 0.09139 & 4.502 \\
$A_{\text {land }}$ & 210 & 0.5434 & 0.4937 & 811.206 & 0.10497 & 2.724 \\
\hline
\end{tabular}

The layouts corresponding to the optimal solutions are presented in Fig. 5 with the annual performance of each heliostat in the field; furthermore, the development of the fields in the four cardinal direction is reported in Table 6. Comparing the configuration that maximize the efficiency (design (a) and annual (b)), it is underlined that the design period ( $21^{\text {st }}$ March noon or the entire annual) influences the best heliostat ranking, since the optimal radial spacing are the same. Fig. 5a and 5b demonstrate that large fields are less sensitive to the heliostat position and different layouts have very similar performance. With the economic optimization the layout results more expanded in every direction: the transition radii between the group are closer to the tower, due to a selected lower THT, and most of the heliostats are in the third group. The transition between the second and the third group is clearer at $R=662$ $\mathrm{m}$, mainly due to a sudden change in efficiency, especially in the north region. This behavior is emphasized when the solution that minimize the land is observed (Fig. 5d): the mean efficiency of the heliostats in the third group is lower than $42 \%$, justifying the penalization 
of the annual performance. The analysis of the land occupancy shows that for the minimization of the area the expansion along the four direction is uniform, although a gap is observed in field in the south region of the third group, where the $f_{s b}$ has the worst values.

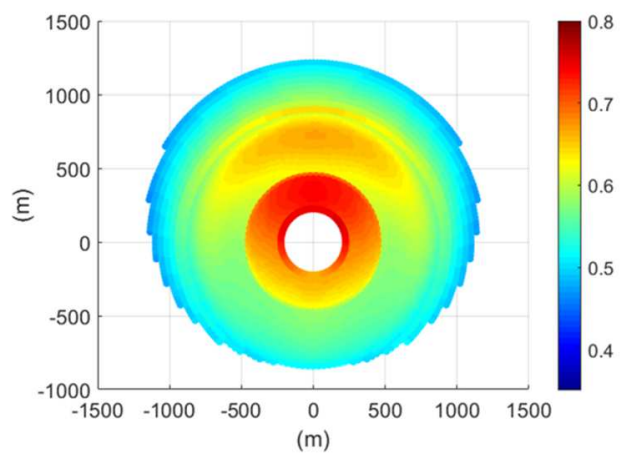

(a) $\max \left(\eta_{\text {des }}\right)$

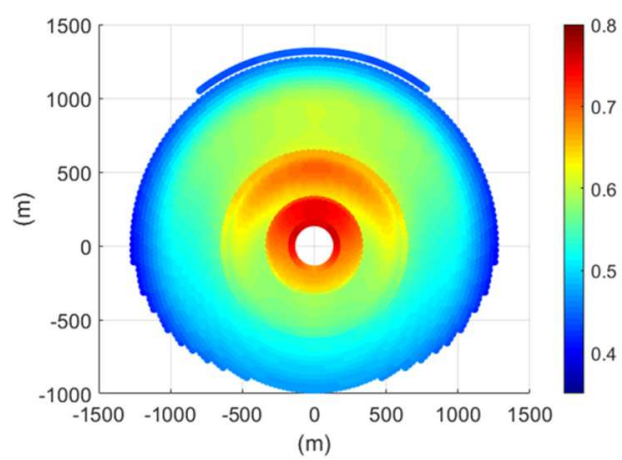

(c) $\min (L C O E)$

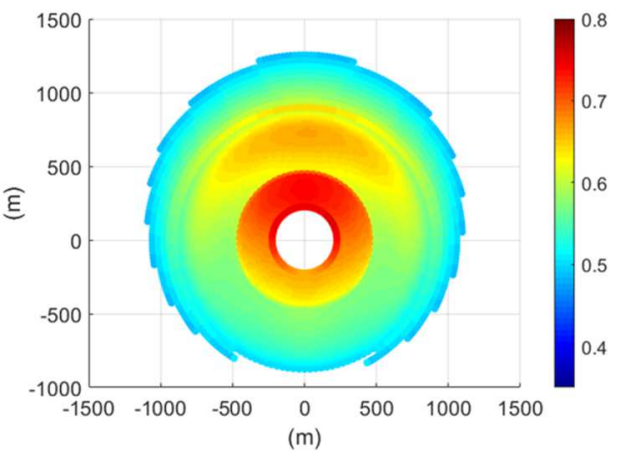

(b) $\max \left(\eta_{\text {ann }}\right)$

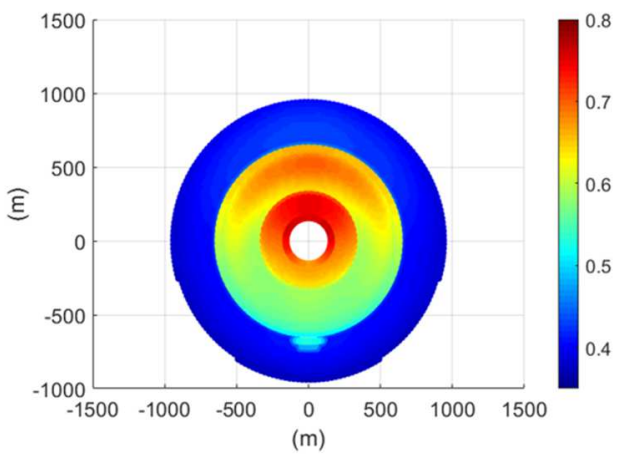

(d) $\min \left(A_{\text {land }}\right)$

Fig. 5. Layout configuration corresponding to the different objective function.

Table 6. Summary of the optimal layouts.

\begin{tabular}{ccccc}
\hline & \multicolumn{4}{c}{ Optimal layouts } \\
\cline { 2 - 5 } & $\max \left(\eta_{\text {des }}\right)$ & $\max \left(\eta_{\text {ann }}\right)$ & $\min (\mathrm{LCOE})$ & $\min \left(A_{\text {land }}\right)$ \\
\hline$R_{N}$ & 1214 & 1256 & 1319 & 936 \\
$R_{S}$ & 842 & 876 & 976 & 936 \\
$R_{E-W}$ & 1136 & 1060 & 1260 & 936 \\
\hline
\end{tabular}

\section{Conclusion}

The paper deals with a detailed analysis of a large-scale heliostat field for a solar tower plant of 100 MWe. The model developed, with a Matlab procedure, can simulate the field performance with a good accuracy and a limited computational demand. Different approaches have been proposed for the design of the field and the influence of the objective function on the performance is evaluated.

The optimal radial spacing is defined as function of the tower height, increasing the optical height of the target the field is expanded when the efficiency is maximized. The field efficiency when the number of heliostats become huge results less influenced by the individual position of the mirrors and different layouts have the same performance: it is possible to reduce the design period to the solar noon of the $21^{\text {st }}$ March and maximize the 
annual efficiency. The efficiency trend is increasing with tower height, however, when the tower is greater than $210 \mathrm{~m}$ the gain is limited to 0.3 percent.

When the economic function is considered, the cost influences the optimal solution and a clear minimum is identified for a tower height of $210 \mathrm{~m}$. The resulting layout is less efficient and more expanded but the LCOE is reduce by more than $5 \%$.

Lastly, minimizing the radial spacing in the field allows to minimize the occupied land ($25 \%$ or $-40 \%$ with respect to different plant optimization) but the efficiency is high penalized and the electricity cost is increased by more than 1 cent $(+13 \%)$.

The four objective function leads to quite different layout and the selected strategy strongly influences the performance. Future work will take care about the matter of receiver sizing, limiting the spillage efficiency and respecting the limit energy flux of the materials and the purpose of the authors is to extend the competitiveness of central receiver system to small scale application, for a widespread diffusion of the tower technology.

\section{References}

1. E. Dall, G. H. Muller, F. Bailey, R. Jagau, A. R. Pfohl, J. Swart, and J. M. S. Caballos, AIP Conf. Proc. 2126, (2019)

2. K. R. Bhargav, F. Gross, and P. Schramek, Energy Procedia 49, 40 (2013)

3. M. Gadalla and M. Saghafifar, Int. J. Energy Res. 42, 3145 (2018)

4. G. Brumana, G. Franchini, and E. Ghirardi, Energy Procedia 148, 615 (2018)

5. J. Coventry and J. Pye, Energy Procedia 49, 60 (2013)

6. R. Chen, Z. Rao, and S. Liao, Energy Convers. Manag. 177, 385 (2018)

7. M. Gadalla and M. Saghafifar, Sol. Energy 136, 197 (2016)

8. Y. Luo, T. Lu, and X. Du, Energy Convers. Manag. 177, 682 (2018)

9. P. Schöttl, S. Rohani, E. Leonardi, L. Pisani, I. Les, A. Mutuberria, and P. Nitz, AIP Conf. Proc. 2126, 030053 (2019)

10. C. Li, R. Zhai, H. Liu, Y. Yang, and H. Wu, Appl. Therm. Eng. 128, 33 (2018)

11. E. Leonardi, L. Pisani, I. Les, A. M. Larrayoz, S. Rohani, and P. Schöttl, Sol. Energy 180, 601 (2019)

12. S. Kiwan and S. Al Hamad, J. Sol. Energy Eng. 141, 011010 (2019)

13. N. C. Cruz, S. Salhi, J. L. Redondo, J. D. Álvarez, M. Berenguel, and P. M. Ortigosa, J. Supercomput. 75, 1 (2018)

14. F. J. Collado and J. Guallar, Energy 178, 115 (2019)

15. L. L. Vant-Hull, J. Sol. Energy Eng. 124, 165 (2002)

16. J. Wang, L. Duan, Y. Yang, and L. Yang, Sol. Energy 180, 456 (2019)

17. F. J. Collado and J. Guallar, SolarPACES 2017 210002, (2018)

18. B. L. Kistler, A User's Manual for DELSOL3: A Computer Code for Calculating the Optical Performance and Optimal System Design for Solar Thermal Central Receiver Plants. (1986)

19. M. Sánchez and M. Romero, Sol. Energy 80, 861 (2006)

20. J. Ballestrín and A. Marzo, Sol. Energy 86, 388 (2012)

21. F. J. Collado and J. Guallar, Sol. Energy 169, 556 (2018)

22. S. M. Besarati and D. Yogi Goswami, Renew. Energy 69, 226 (2014)

23. G. Sassi, Sol. Energy 31, 331 (1983)

24. C. S. Turchi and G. A. Heath, (2013)

25. F. J. Collado and J. Guallar, Renew. Sustain. Energy Rev. 20, 142 (2013)

26. J. Remund, S. Müller, S. Kunz, and C. Schilter, Meteonorm Handbook Part II: Theory (2012)

27. J. Wang, L. Duan, and Y. Yang, Energy 155, 15 (2018) 